\title{
B-Cell Lymphoblastic Lymphoma in Relapse Presenting as a Uterine Mass: A Case Report and Review of Literature
}

\author{
Rim Abou Chakra ${ }^{a}$ Bernard Najib ${ }^{a}$ Wael Abdallah ${ }^{a} \quad$ Mira Akiki $^{b}$ \\ Lea El Khoury ${ }^{b}$ Ali Atoui $^{c}$ Malak Moubarak $^{a}$ David Atallah $^{a}$ \\ aDepartment of Gynecologic Oncology and Reproductive Medicine, Hotel-Dieu de France \\ University Hospital, Saint-Joseph University of Beirut, Beirut, Lebanon; bepartment of \\ Pathology, Hotel-Dieu de France University Hospital, Saint-Joseph University of Beirut, \\ Beirut, Lebanon; 'Hematology-Oncology Division, Internal Medicine Department, American \\ University of Beirut Medical Center, Beirut, Lebanon
}

\section{Keywords}

Lymphoblastic lymphoma $\cdot$ Leiomyosarcoma $\cdot$ Uterine mass

\begin{abstract}
B-cell lymphoblastic lymphoma (LBL) is a highly aggressive malignant proliferation of lymphoblasts of B-origin grouped with acute lymphoblastic leukemia. Multiple studies demonstrated the various sites of involvement in adult LBL. The involvement of the uterus as a site of relapse for such disease is rare. We herein report the case of relapsed B-cell LBL mimicking endometrial sarcoma. The patient is a 56-year-old female patient known to have B-cell LBL on chemotherapy. She presented with abdominal pain and fever. Positron emission tomodensitometry-computed tomography showed the presence of a uterine mass with bilateral iliac lymph node involvement. She underwent surgery with mass removal and pathology showed relapsed B-cell LBL.
\end{abstract}

(c) 2021 The Author(s).

Published by S. Karger AG, Basel

\section{Introduction}

Lymphoblastic leukemias/lymphomas are neoplasms of precursor T cells and B cells or lymphoblasts. A diagnosis of lymphoblastic lymphoma (LBL) is made when the disease is limited to primary nodal and extranodal sites with minimal involvement of bone marrow $(<20 \%$ lymphoblasts in marrow). Combination chemotherapy is a standard treatment to LBL, but relapse is common. The involvement of the uterus as a site of relapse for such disease is rare. We herein report the case of relapsed B-cell LBL mimicking endometrial sarcoma. 


\section{Case Reports in Oncology}
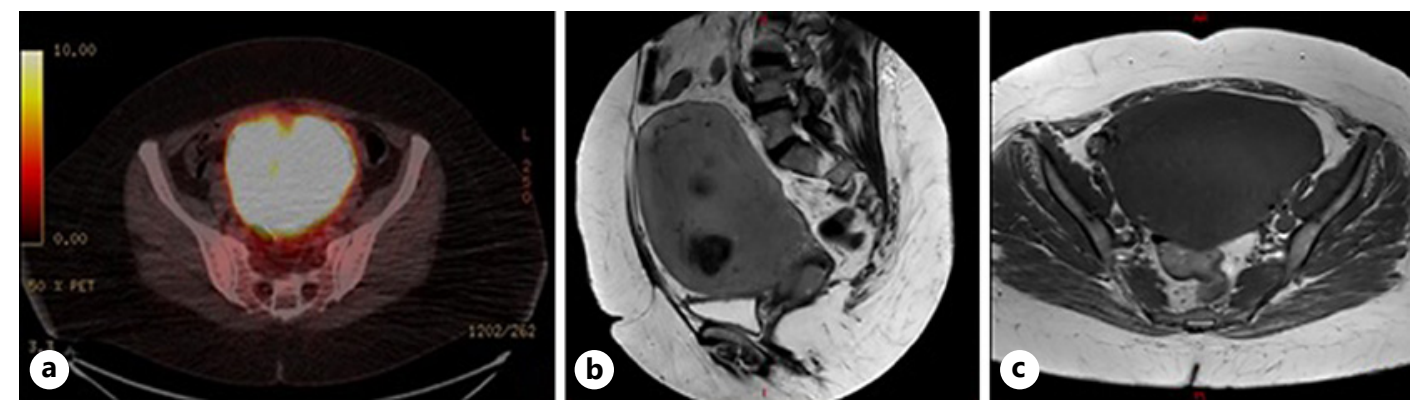

Fig. 1. a PET-CT scan showing diffuse increase uptake in the uterus. b A sagittal plane of a MRI with gadolinium showing the mass of $15 \mathrm{~cm}$ described as possible leiomyosarcoma of the uterine cavity and a second mass in the anterior aspect of the cervix measuring $3.8 \mathrm{~cm}$ with 2 suspicious lymph nodes of 1.3 and $1.2 \mathrm{~cm}$ noted in the right and left iliac chains respectively. c A transverse plane showing dense adherence of the mass to the sigmoid. PET, positron emission tomodensitometry-computed tomography.

\section{Case Presentation}

It is a case of a 56-year-old lady, Gravida 3 Para 3, in menopause, not on hormonal replacement therapy who presented with bilateral flank pain, constipation, and obstipation. The patient has a history of B-cell acute LBL initially diagnosed 1 year ago on a clavicular lesion biopsy after she presented with a history of osteitis resistant to antibiotics. Bone marrow at diagnosis showed no involvement by lymphoma. After 2 cycles of R-Hyper CVAD (rituximab, cyclophosphamide, vincristine, adriamycin, and dexamethasone) a positron emission tomodensitometry (PET) scan was performed and showed complete remission. After receiving a total of 8 cycles of chemotherapy, a control PET scan showed an increase in the size of the uterus measuring $12.5 \mathrm{~cm}$ in the medial-lateral aspect with increased FDG uptake of the uterus and the cervix (shown in Fig. 1a). A bone marrow exam was performed and showed $50-60 \%$ cellular marrow with maturing trilineage hematopoiesis and $<1 \%$ blasts. At this moment, the patient was asymptomatic and the physical examination revealed a palpable pelvic mass with anon-tender abdomen. She has no vaginal bleeding or discharge. She has no family history of gynecologic cancer. Pap smear was normal. MRI with gadolinium was done for better characterization of the mass and showed further increase in the size of the uterine mass in 1 month reaching $15 \mathrm{~cm}$ described as possible leiomyosarcoma of the uterine cavity. A second mass was noted in the anterior aspect of the cervix measuring $3.8 \mathrm{~cm}$ with 2 suspicious lymph nodes of 1.3 and $1.2 \mathrm{~cm}$ noted in the right and left iliac chains, respectively (shown in Fig. 1b, c).

After 1 month, she presented to the emergency room with acute and persistent flank pain and obstipation for $24 \mathrm{~h}$, a computed tomography scanner of the abdomen and pelvis with intravenous contrast was performed and showed the same findings of a heterogeneous uterine mass, an enlarged uterus compressing the sigmoid colon associated with colonic dilatation. There was evidence of bilateral hydronephrosis with large bowel obstruction. Multiple bilateral enlarged lymph nodes were noted in the external iliac chain, the largest measuring $1.8 \times 1.6 \mathrm{~cm}$.

Taking into consideration the aspect of the mass seen on MRI described as possible leiomyosarcoma and the patient's conditions, she was admitted for surgical removal. Bilateral double J was inserted, and then she underwent an emergent exploratory laparotomy: a $15 \mathrm{~cm}$ uterine mass was adherent to the sigmoid. Total abdominal hysterectomy with bilateral salpingo-oophorectomy with sigmoidectomy and a colorectal anastomosis (posterior pelvic 


\section{Case Reports in Oncology}

Case Rep Oncol 2021;14:868-873

\begin{tabular}{l|l}
\hline DOI: 10.1159/000515196 & ( 2021 The Author(s). Published by S. Karger AG, Basel
\end{tabular} www.karger.com/cro

Abou Chakra et al.: Uterine Involvement in Lymphoblastic Lymphoma
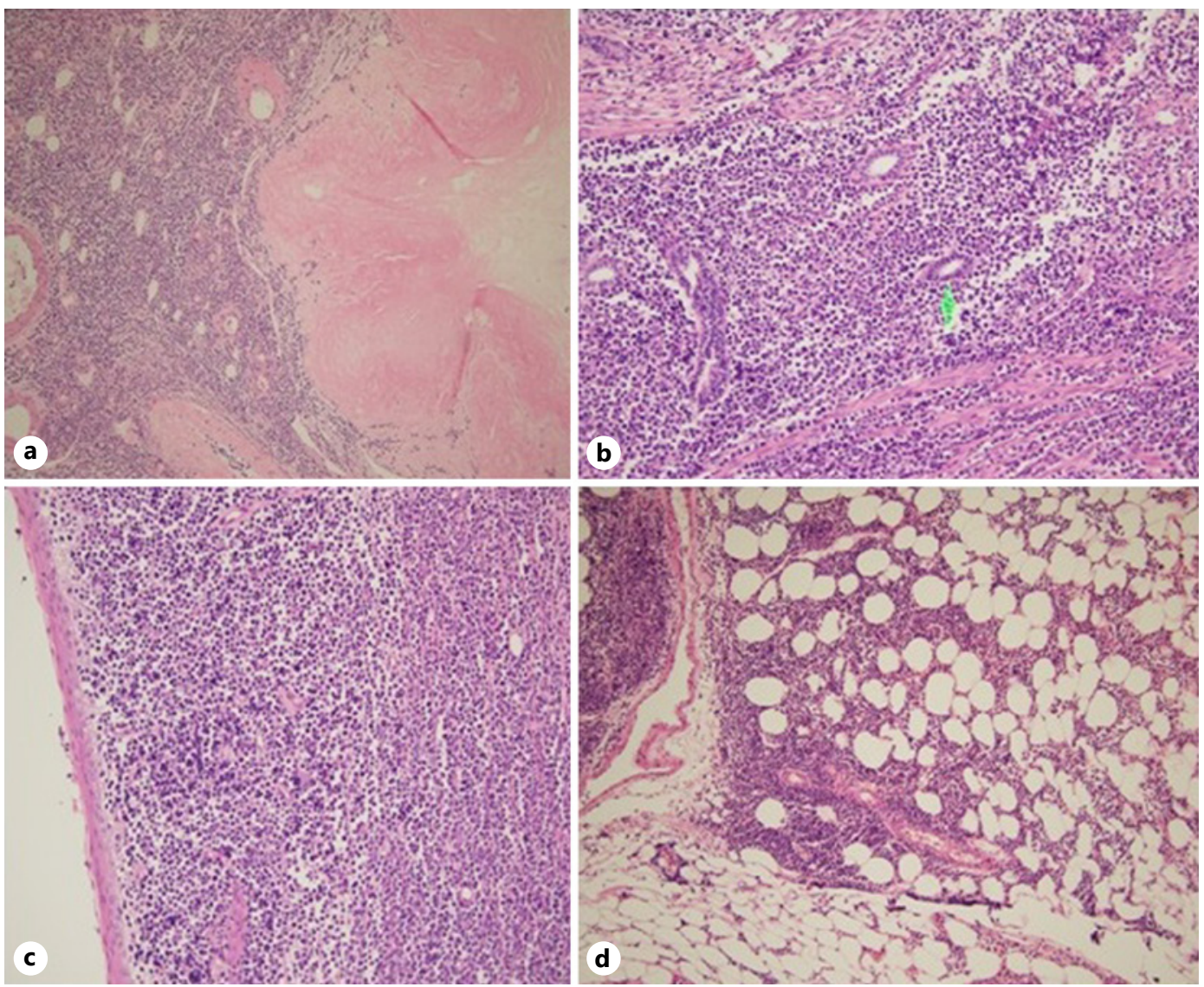

Fig. 2. Hematoxylin-eosin stain microscopy of the neoplastic lymphocytic proliferation. a Section from the left ovary exhibiting an infiltrated corpus albicans $(\times 10$ magnification). b Endometrial gland diffusely infiltrated by neoplastic lymphocytes ( $\times 20$ magnification). Infiltration of the cervix $(\times 20$ magnification) (c) and of the mesocolon $(\times 10$ magnification) $(\mathbf{d})$.

exenteration) was done en bloc. The specimen was sent for frozen evaluation and showed a relapsed B-cell LBL in the uterine. Based on this result, pelvic lymphadenectomy was not performed as it was not indicated.

Surgical pathology reported a diffuse infiltration of the uterus, the cervix, the ovaries, the fallopian tubes, the parametrium, and the mesocolon by neoplastic lymphocytes disclosing hyperchromatic irregular nuclei. These neoplastic cells infiltrated massively most of the leiomyomas. Immunohistochemistry showed that the neoplastic lymphocytes were diffusely positive for CD45, CD79a, PAX5, Bcl2, and TdT, while negative for cytokeratin C, Desmin, CD3, and CD20. The diagnosis of B cell (CD20 negative) infiltrating the female genital tract was rendered (shown in Fig. 2, 3). The postoperative period was without complications and the patient was discharged on day 8 . She is planned to receive gemcitabine and oxaliplatin chemotherapy for a total of 6 cycles.

The authors state that they have followed the principles outlined in the Declaration of Helsinki for all human or animal experimental investigations. In addition, written informed consent was obtained from the patient for publication of this case report and any accompanying images. 


\section{Case Reports in Oncology}

Case Rep Oncol 2021;14:868-873

\begin{tabular}{l|l}
\hline DOI: $10.1159 / 000515196$ & @ 2021 The Author(s). Published by S. Karger AG, Basel
\end{tabular} www.karger.com/cro

Abou Chakra et al.: Uterine Involvement in Lymphoblastic Lymphoma
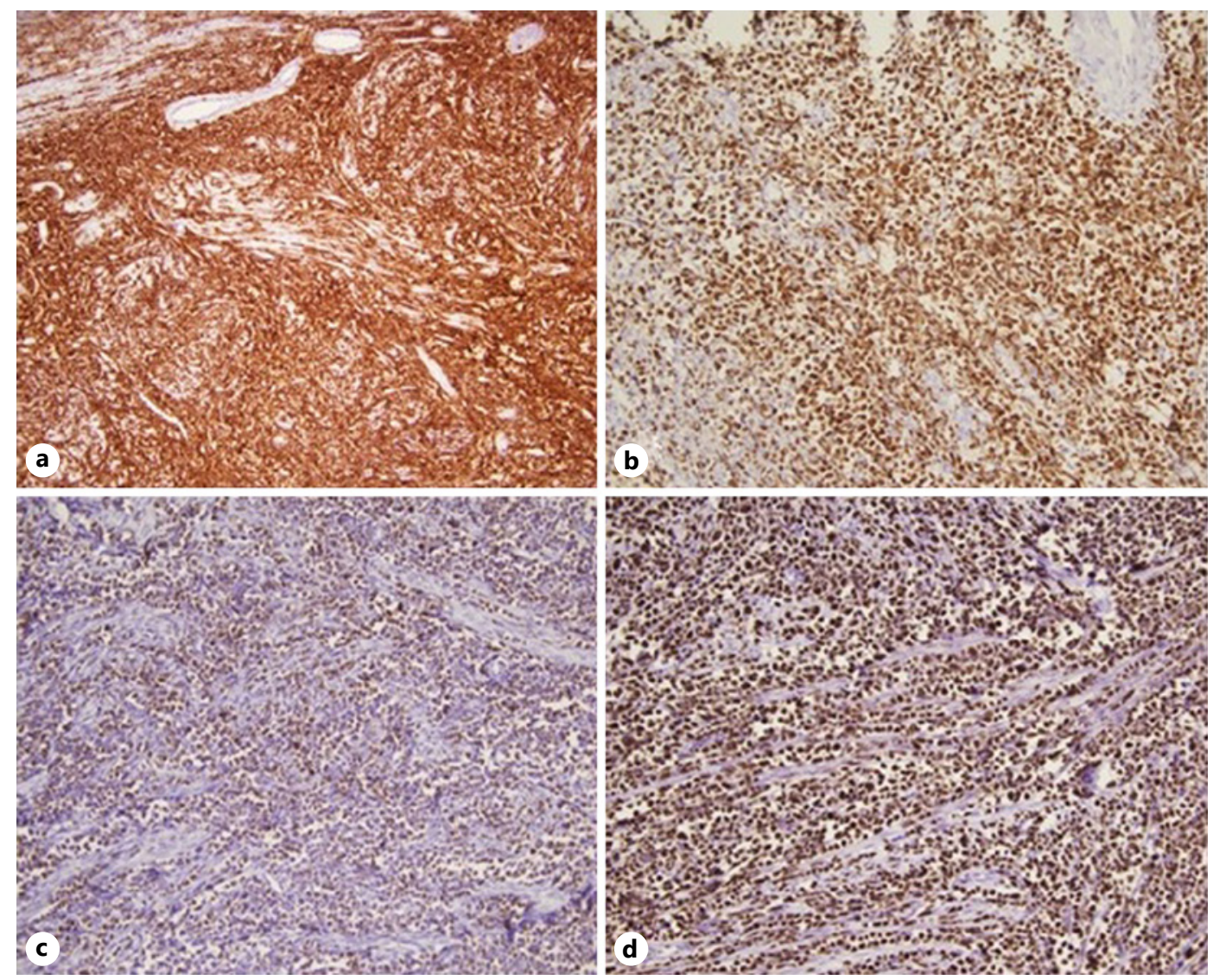

Fig. 3. Immunochemistry of the B-cell LBL. The neoplastic cells are positive for CD45 (a), TdT (b), CD79a (c), and PAX5 (d). LBL, lymphoblastic lymphoma.

\section{Discussion}

In this case report, we report a case of B-cell LBL involving the uterus presenting similarly to locally advanced endometrial leiomyosarcoma. Multiple studies have demonstrated the distribution of extramedullary sites involved among patients with LBL. These sites were mainly the pleura, pericardium, central nervous system, liver and spleen, and to a lesser extent the skin and kidneys [1]. However, the involvement of the female genital tract is rare and accounts for $<1 \%$ of extranodal lymphoma and/or leukemia [2].

Patients with hematological malignancies involving the genital tract usually present with symptoms of vaginal bleeding, abdominal pain due to a pelvic mass along with other constitutional symptoms such as B symptoms (weight loss, night sweats, and fever). Our patient presented with nonspecific diffuse abdominal pain and obstipation without any other signs or symptoms with imaging showing a uterine mass.

The appearance of the mass on imaging favored leiomyosarcoma (LMS). LMS is usually solitary, heterogeneous, and poorly demarcated masses on MRI [3]. They frequently show areas of high intensity on T1-weighted images corresponding to necrosis. T2-weighted images show intermediate to high signal. After intravenous gadolinium contrast, LMS demonstrates early and heterogeneous enhancement [3-5]. PET scan is sensitive to detect tumors with a high rate of proliferation. It is sensitive for staging high-grade non-Hodgkin lymphoma [6]. However, in the setting of a single uterine mass, PET scan is not sensitive to differentiate between LBL and LMS as both entities will be highly FDG avid. Taking into consideration the 
history of the patient and despite the rare involvement of the genital tract by hematological malignancies and the typical features of leiomyosarcoma on MRI, one should always suspect a relapse of the primary disease.

In this patient, our decision to perform surgery was based on the high specificity and diagnostic accuracy of the MRI in detecting uterine sarcomas [7]. In this situation, according to the national comprehensive cancer network NCCN guidelines, preoperative imaging and biopsy may help to identify uterine sarcomas although biopsy sensitivity is less than for endometrial cancer. The suspicion of malignant mesenchymal sarcoma is a contraindication of fragmentation and morcellation. Hence, a mass biopsy was not done during the laparotomy, and a surgical removal en bloc was performed. Pathological findings of the surgical specimen showed relapse B-LBL. Histopathological features of LBL show usually a diffuse proliferation of lymphoblasts comparable to acute lymphoblastic leukemia cells [8]. Immunohistochemistry will confirm the B-cell origin.

In summary, B-cell LBL is aggressive hematological neoplasms and extramedullary relapses of the disease are frequent. When the patient is symptomatic, imaging is necessary as relapses may occur at multiple sites without bone marrow involvement. Interpretation of radiologic findings should always be correlated to the clinical presentation as these findings alone are nonspecific. In the context of known hematological malignancy namely LBL, any soft tissue mass can represent infection, secondary neoplasms, or relapse of primary disease [9].

\section{Statement of Ethics}

The authors state that they have followed the principles outlined in the Declaration of Helsinki for all human or animal experimental investigations. In addition, written informed consent was obtained from the patient for publication of this case report and any accompanying images.

\section{Conflict of Interest Statement}

The authors have no conflicts of interest to declare.

\section{Funding Sources}

The authors did not receive any funding sources.

\section{Author Contributions}

All the authors have contributed equally in the literature search and redaction of this article.

\section{References}

1 Sun L, Friedman AH, Rodgers R, Schear M, Greaves G, Freidl KB. T-cell lymphoblastic lymphoma involving the ocular adnexa: report of two cases and review of the current literature. Orbit. 2019;38(5):412-8.

2 Lyman MD, Neuhauser TS. Precursor T-cell acute lymphoblastic leukemia/lymphoma involving the uterine cervix, myometrium, endometrium, and appendix. Ann Diagn Pathol. 2002;6(2):125-8.

\section{Karger'}


3 Tamai K, Koyama T, Saga T, Morisawa N, Fujimoto K, Mikami Y, et al. The utility of diffusion-weighted MR imaging for differentiating uterine sarcomas from benign leiomyomas. Eur Radiol. 2008;18(4):723-30.

4 Tanaka YO, Nishida M, Tsunoda H, Okamoto Y, Yoshikawa H. Smooth muscle tumors of uncertain malignant potential and leiomyosarcomas of the uterus: MR findings. J Magn Reson Imaging. 2004;20(6):998-1007.

5 Lin G, Yang LY, Huang YT, Ng KK, Ng SH, Ueng SH, et al. Comparison of the diagnostic accuracy of contrastenhanced MRI and diffusion-weighted MRI in the differentiation between uterine leiomyosarcoma/smooth muscle tumor with uncertain malignant potential and benign leiomyoma. J Magn Reson Imaging. 2016;43(2): 333-42.

6 Ngeow JYY, Quek RHH, Ng DCE, Hee SW, Tao M, Lim LC, et al. High SUV uptake on FDG-PET/CT predicts for an aggressive B-cell lymphoma in a prospective study of primary FDG-PET/CT staging in lymphoma. Ann Oncol. 2009;20(9):1543-7.

7 Lin G, Yang L-Y, Huang Y-T, Ng K-K, Ng S-H, Ueng S-H, et al. Comparison of the diagnostic accuracy of contrastenhanced MRI and diffusion-weighted MRI in the differentiation between uterine leiomyosarcoma/smooth muscle tumor with uncertain malignant potential and benign leiomyoma. J Magn Reson Imaging. 2016 Feb; 43(2):333-42.

8 Swerdlow SH, Campo E, Pileri SA, Harris NL, Stein H, Siebert R, et al. The 2016 revision of the World Health Organization classification of lymphoid neoplasms. Blood. 2016;127(20):2375-90.

9 Arrigan M, Smyth L, Harmon M, Flynn C, Sheehy N. Imaging findings in recurrent extramedullary leukaemias. Cancer Imaging. 2013;13(1):26-35. 